\title{
Attachment-induced ionization instability in electronegative capacitive RF discharges
}

\author{
A Descoeudres, L Sansonnens and Ch Hollenstein \\ Centre de Recherches en Physique des Plasmas, Ecole Polytechnique Fédérale de \\ Lausanne, CH-1015 Lausanne, Switzerland \\ E-mail: antoine.descoeudres@epfl.ch
}

\begin{abstract}
Attachment-induced ionization instability has been experimentally observed in $\mathrm{O}_{2}$ and $\mathrm{CF}_{4}$ capacitive $\mathrm{RF}$ discharges using time-resolved voltage probe, Langmuir probe, optical emission and mass spectrometry measurements. This instability occurs under specific conditions of power and pressure, and produces synchronized oscillations in the $\mathrm{kHz}$ range on potentials, emission intensity and positive ions fluxes. On the contrary, the $\mathrm{SF}_{6}$ plasma was observed to remain stable under all experimental conditions. This can be understood by considering attachment and ionization cross sections of these gases and applying the theoretical criterion of instability. Contrary to $\mathrm{O}_{2}$ and $\mathrm{CF}_{4}$, the attachment rate coefficient of $\mathrm{SF}_{6}$ is very high at low energy and has a negative dependence on the electronic temperature. The application of the criterion shows clearly that $\mathrm{O}_{2}$ and $\mathrm{CF}_{4}$ plasmas are unstable at low electronic temperature, and that the $\mathrm{SF}_{6}$ plasma is stable due to its particular low-energy attachment cross section.
\end{abstract}

\section{INTRODUCTION}

Radio-frequency discharges in electronegative gases are widely used in industrial processes, particularly in the manufacturing of semiconductor devices. Oxygen $\left(\mathrm{O}_{2}\right)$, sulfur hexafluoride $\left(\mathrm{SF}_{6}\right)$ and carbon tetrafluoride $\left(\mathrm{CF}_{4}\right)$ are the main electronegative gases employed for etching applications [1]. Inductively coupled plasmas (ICP) are used for most electronic devices but capacitively coupled plasmas (CCP) remain the choice in large area applications such as thin film transistors for flat display.

The presence of negative ions in a discharge changes considerably the physics of the plasma. Especially, several instabilities linked with the electronegativity of the gas can develop. Ionization waves have been studied in $\mathrm{SF}_{6} \mathrm{DC}$ glows [2]. In DC glows of other electronegative gases such as $\mathrm{CO}_{2}, \mathrm{CO}$ and $\mathrm{O}_{2}$, the attachment-induced ionization instability has been observed [3, 4, 5] and described in detail in [5]. There are also instabilities in inductive $\mathrm{RF}$ discharges, e.g. in $\mathrm{O}_{2}$ and $\mathrm{SF}_{6}[6,7]$. In this case the instability is attributed to the transition between inductive and capacitive modes.

The attachment-induced ionization instability occurs in DC glow discharges but also in capacitively coupled RF discharges. The instability was first observed in $\mathrm{O}_{2}$ capacitively 
coupled RF plasmas [8] and was also modelled for $\mathrm{CF}_{4}[9,10]$. Nevertheless, the experimental characterization of this phenomenon is incomplete. Particularly, the question of appearance of the instability in other electronegative gases is still open [8]. This can be important for processes that require stable plasma characteristics.

In this paper we present observations of the attachment-induced ionization instability in $\mathrm{O}_{2}$ and $\mathrm{CF}_{4}$ capacitively coupled plasmas. The instability appears as oscillations on all the diagnostics used. The experimental conditions under which the discharge is unstable are also investigated. In the discussion we present the mechanism of the instability and apply the theoretical instability criterion of Nighan and Wiegand [5]. This criterion explains another important experimental result, namely the stability of the $\mathrm{SF}_{6}$ plasma. Combined with simulation results, the criterion can also predict the unstable plasma conditions of the discharge.

\section{EXPERIMENTAL SETUP}

The experimental apparatus shown in figure 1 is a parallel plate capacitively coupled reactor comprising two stainless steel cylindrical electrodes $13 \mathrm{~cm}$ in diameter with a 2.5 or $4 \mathrm{~cm}$ electrode gap (for more details, see [11]). The typical plasma parameters used were: gas pressure 0.1 - 0.4 mbar, gas flow 13 sccm in $\mathrm{SF}_{6}, 20 \mathrm{sccm}$ in $\mathrm{CF}_{4}$ and $50 \mathrm{sccm}$ in $\mathrm{O}_{2}$, power up to $100 \mathrm{~W}$ at $13.56 \mathrm{MHz}$ excitation frequency. A low frequency $(50 \mathrm{~Hz}-1 \mathrm{kHz})$ square wave modulation was applied to the high frequency excitation. This modulation permitted the synchronization of the diagnostics, the measurements of the negative ions during the afterglow [11] and to differentiate between transient and steady-state phenomena.

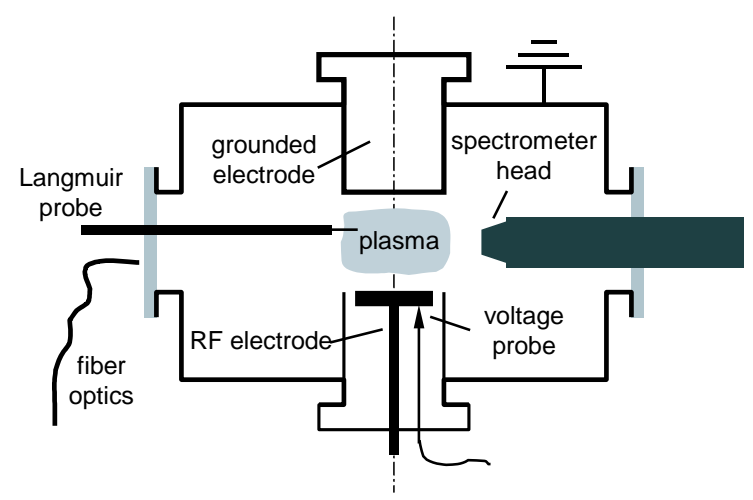

Figure 1. Schematic drawing of the reactor and diagnostics

For the characterization of the plasma, we used a voltage probe connected directly to the RF electrode to measure the external voltage envelope, and consequently the peakto-peak voltage $V_{p p}$ and the self-bias $V_{s b}$. The external envelope is the maximum and the minimum of the voltage measured at the RF electrode, averaged over a large number of low frequency modulation periods. A Scientific Systems Langmuir probe coupled to a 
boxcar integrator was used to obtain time resolved current-voltage characteristics. The total plasma light was focused by a lens and collected with quartz fiber. We measured the global emission intensity with a Perkin Elmer C953 photomultiplier. The voltage and emission intensity data were recorded with a LeCroy digital oscilloscope. Finally, we used a Balzers PPM422 mass spectrometer combined with a EG\&G Ortec multi channel scaler to obtain time-resolved ion flux measurements. The extraction head was biased to $+5 \mathrm{~V}$ to collect a sufficient number of negative ions, no biasing was used when measuring the positive ions.

\section{OBSERVATIONS OF THE INSTABILITY}

An instability was observed both in $\mathrm{O}_{2}$ and $\mathrm{CF}_{4}$ discharges. The instability is not caused by external circuit effects, as we observed it in two different RF reactors, with two different RF matchboxes and amplifiers. The instability behaves in the same general way in these two plasmas. On the contrary, $\mathrm{SF}_{6}$ discharges remain stable in all experimental conditions.

Oscillations are seen on the external voltage envelope. These oscillations develop under specific conditions of power and pressure. Figures 2 and 3 show voltage envelopes in $\mathrm{O}_{2}$ discharges as a function of $V_{p p}$ and pressure respectively. The threshold values of the unstable zone are different in $\mathrm{O}_{2}$ and $\mathrm{CF}_{4}$, but both plasmas behave generally in the same way. At low power the discharge is stable, becomes unstable with increasing power, and is stable again at high RF power. At 0.25 mbar, the instability occurs for applied peak-to-peak voltage between $185 \mathrm{~V}$ and $480 \mathrm{~V}$ in $\mathrm{O}_{2}$ discharges, and between $220 \mathrm{~V}$ and $550 \mathrm{~V}$ in $\mathrm{CF}_{4}$ discharges. A pressure threshold was also observed for the appearance of the instability. The plasma remains stable below 0.195 mbar in $\mathrm{O}_{2}$ and 0.1 mbar in $\mathrm{CF}_{4}$ at $V_{p p} 250 \mathrm{~V}$. Our measurements of the unstable zone for $\mathrm{O}_{2}$ plasma are in good agreement with the results of Katsch et al [8], who found the unstable zone around 0.3 mbar and $300 \mathrm{~V}$. We observed that the inter-electrode gap and gas flux have no influence on the presence of the instability.
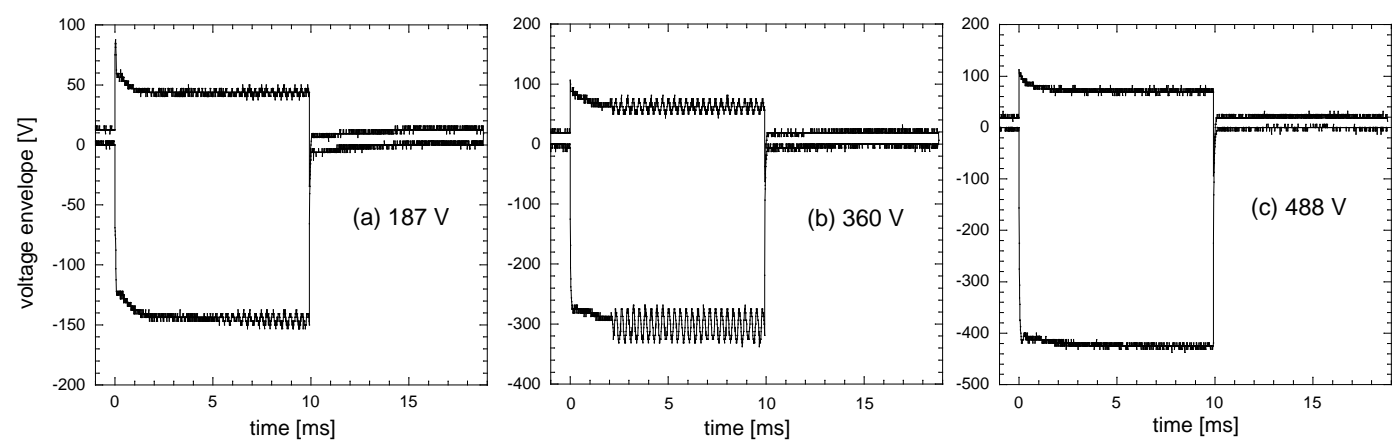

Figure 2. Instability on voltage envelope in $\mathrm{O}_{2}$ plasma as a function of $V_{p p}(0.25$ mbar, $50 \mathrm{~Hz}$ modulation) : (a) $187 \mathrm{~V}$ (b) $360 \mathrm{~V}$ (c) $488 \mathrm{~V}$ 

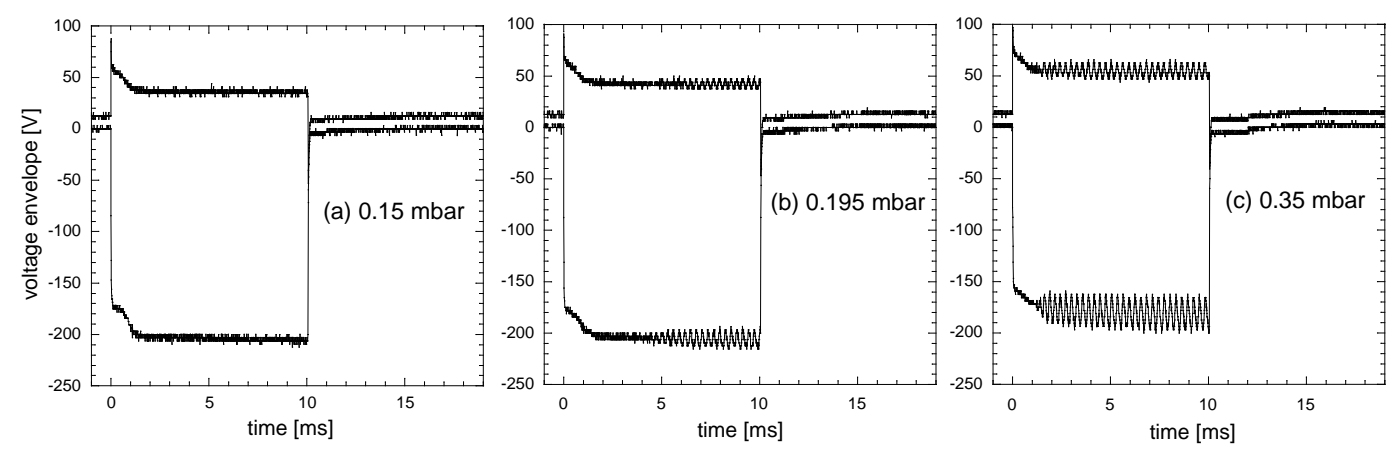

Figure 3. Instability on voltage envelope in $\mathrm{O}_{2}$ plasma as a function of pressure (250 $\mathrm{V} V_{p p}, 50 \mathrm{~Hz}$ modulation) : (a) 0.15 mbar (b) 0.195 mbar (c) 0.35 mbar

We see in figures 2 and 3 that it takes a few ms for the oscillations to appear, and then they remain constant. The frequency of the oscillations is in the $\mathrm{kHz}$ range: $3.5 \mathrm{kHz}$ in $\mathrm{O}_{2}$ (consistent with results of [8]) and around $80 \mathrm{kHz}$ in $\mathrm{CF}_{4}$ at 0.25 mbar. In fact, the instability can also be observed without power modulation (continuous wave discharge). Since the voltage envelopes measurements are averaged over many low frequency square wave cycles, the instability is a very reproducible and regular phenomenon.

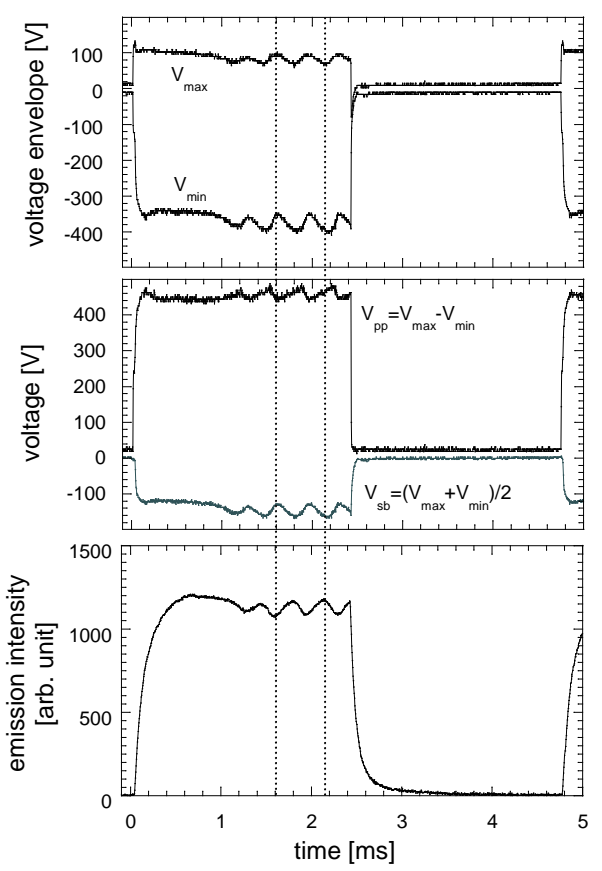

Figure 4. Instability on voltage envelope along with peak-to-peak voltage, self-bias voltage and global emission intensity in $\mathrm{O}_{2}$ plasma $\left(0.25\right.$ mbar, $450 \mathrm{~V} V_{p p}, 210 \mathrm{~Hz}$ modulation)

The different diagnostics show that many physical properties of the plasma follow the oscillations observed on voltage envelopes. Figure 4 shows variations of $V_{p p}, V_{s b}$ and of the global emission intensity in a $\mathrm{O}_{2}$ plasma. The fluxes of the positive ions $\mathrm{CF}_{3}^{+}$in pure 
$\mathrm{CF}_{4}$ plasma and $\mathrm{O}_{2}^{+}$in pure $\mathrm{O}_{2}$ plasma at various energies are also affected, as shown in figure 5. The voltage oscillations, emission intensity and the ion fluxes were perfectly synchronized. Finally, the Langmuir probe results seem to show oscillations on the floating potential $V_{f}$, plasma potential and electronic saturation current, as Katsch et al reported it for oxygen [8]. We verified that the floating potential was really oscillating by connecting the Langmuir probe (without bias) directly to the oscilloscope.
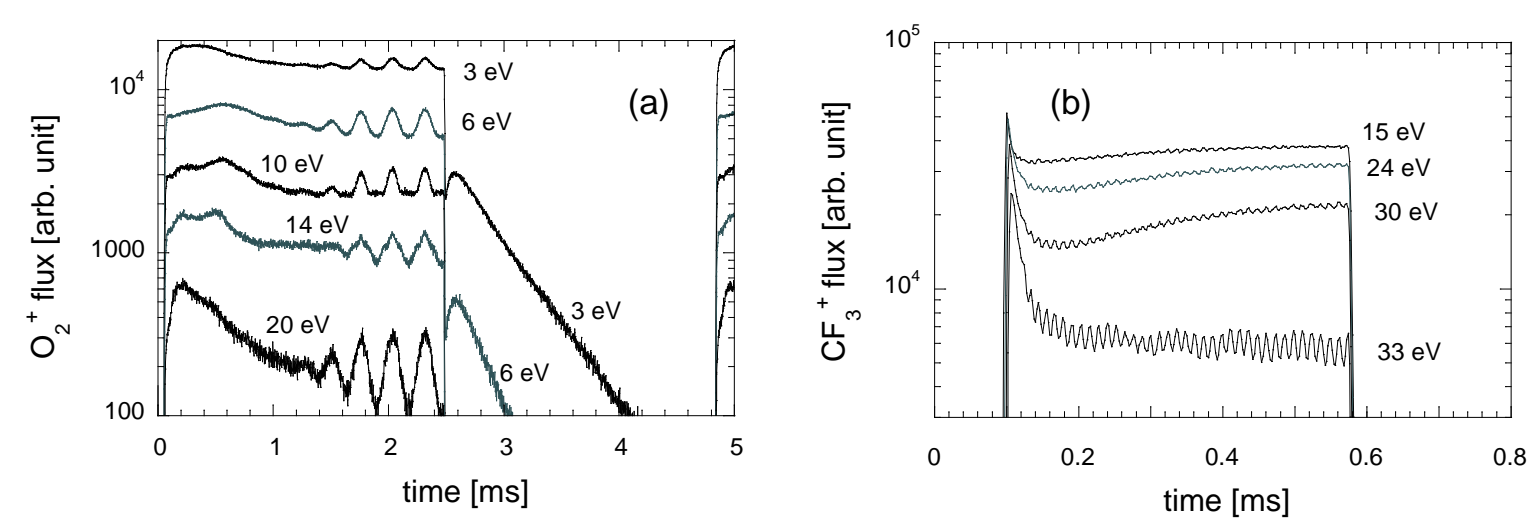

Figure 5. Instability on fluxes of positive ions (a) $\mathrm{O}_{2}^{+}\left(0.25 \mathrm{mbar}, 230 \mathrm{~V} V_{p p}, 210 \mathrm{~Hz}\right.$ modulation) and (b) $\mathrm{CF}_{3}^{+}\left(0.25\right.$ mbar, $200 \mathrm{~V} V_{p p}, 1 \mathrm{kHz}$ modulation)

The amplitude of the oscillations in diluted $\mathrm{Ar}+\mathrm{CF}_{4}$ and $\mathrm{Ar}+\mathrm{O}_{2}$ discharges decreases with increasing concentrations of argon at fixed power and pressure. In $\mathrm{Ar}+\mathrm{CF}_{4}$ discharges for example, the negative ion flux and the amplitude of the oscillations decrease strongly above $80 \%$ of argon. This indicates that negative ions play a crucial part in the development of this type of instability.

\section{DISCUSSION}

The oscillations described previously can be attributed to the attachment-induced ionization instability. The instability mechanism is described in detail by Nigham and Wiegand [5] and can be summarized as follows.

Let us assume a small initial increase in the electronic density $n_{e}$. This will generally be followed by a decrease in the electronic temperature $T_{e}$ due to the quasi-steady nature of the electron energy response [5]. This diminution of $T_{e}$ has different consequences according to the nature of the gas.

In electropositive plasmas, electrons and positive ions are created by ionization and lost mainly by diffusion to the walls and recombination. Ionization decreases if $T_{e}$ is diminished. Thus the electronic density decreases in response to the initial increase in $n_{e}$. This negative feedback stabilizes the perturbation.

In electronegative plasmas, electrons are also lost by attachment, and the efficiency of this process depends on electronic temperature. If the attachment decreases less strongly than the ionization decreases with the diminution of $T_{e}$, the electronic density 
will decrease and the perturbation is stabilized. But in certain electronegative gases, the attachment can decrease more strongly than the decrease in ionization if $T_{e}$ decreases. In this case, the decrease of $T_{e}$ following an increase in $n_{e}$ will cause a further increase of $n_{e}$ (positive feedback), and the discharge becomes unstable. In this type of instability, $n_{e}$ and $T_{e}$ are the physical values that are the source of the oscillations. Their variations lead to changes in the other measured quantities such as the ions fluxes, the emission intensity and the electrical potentials $\left(V_{p p}, V_{s b}, V_{f}, V_{p}\right)$. The plasma must reach certain conditions of $n_{e}$ and $T_{e}$ before the instability can develop. This is the cause of the delay of few ms observed.

This general description has been expressed by a necessary condition for the appearance of the instability by Nigham and Wiegand [5]. The physical basis of this criterion are the conservation equations of electronic and ionic densities. The plasma can be unstable if

$$
R \doteq \frac{\partial k_{a} / \partial T_{e}}{\partial k_{i} / \partial T_{e}}>1
$$

where $k_{a}$ is the attachment rate coefficient and $k_{i}$ the ionization rate coefficient. The rate coefficient $k_{j}$ of the process $j$ is defined by

$$
k_{j} \doteq \sqrt{\frac{2 e}{m_{e}}} \int_{0}^{\infty} \sigma_{j}(E) \cdot f(E) \cdot E \cdot \mathrm{d} E
$$

where $E$ is the energy of the colliding electron, charge $e$ and mass $m_{e}, f$ the energy distribution and $\sigma_{j}$ the cross section of the process.

Since the electron energy distribution and thus the rate coefficients $k_{j}$ are functions of $T_{e}$, the ratio $R$ depends on $T_{e}$ also. In practice, the criterion (1) is fulfilled if the attachment rate coefficient increases with electronic temperature faster than the ionization rate. From a detailed analysis of the electron continuity equation, Nighan and Wiegand also deduce that the negative ion density has to be of the same order as the electron density, otherwise the perturbation is stabilized [5]. In fact the instability involves coupled fluctuations of both negative populations.

For the calculation of the integrals (2), we use the attachment and ionization cross sections shown in figure 6 , given in $[12,13,14,15]$. For the electron energy distributions, we used solutions of the Boltzmann equation calculated with Bolsig from Kinema Software [16]. The calculated distribution remains close to the Maxwellian distribution, and therefore we use the associated Maxwellian electron temperature in the following discussion. Calculated attachment and ionization rate coefficients for $\mathrm{O}_{2}, \mathrm{CF}_{4}$ and $\mathrm{SF}_{6}$ are shown in figure 7 , and the ratio $R$ in figure 8 .

The ionization cross sections and therefore the ionization rate coefficients are quite similar for the three gases. As mentioned above, the ionization rate coefficient decreases with a diminution of the electronic temperature $\left(k_{i}\right.$ is an increasing function of $\left.T_{e}\right)$. But $\mathrm{SF}_{6}$ differs from $\mathrm{O}_{2}$ and $\mathrm{CF}_{4}$ by its attachment cross section. The calculations of $R$ in figure 8 demonstrate that $\mathrm{O}_{2}$ and $\mathrm{CF}_{4}$ plasmas can be unstable at low electronic temperature (under $3.9 \mathrm{eV}$ and $5.05 \mathrm{eV}$ respectively). These gases have such properties 


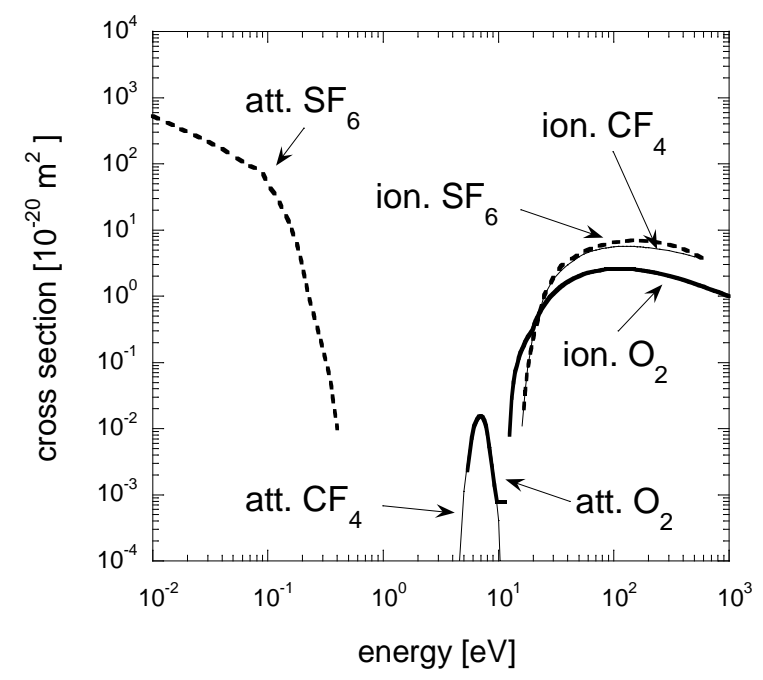

Figure 6. Attachment and ionization cross sections for $\mathrm{O}_{2}[12], \mathrm{CF}_{4}[13,14]$ and $\mathrm{SF}_{6}$ $[15]$

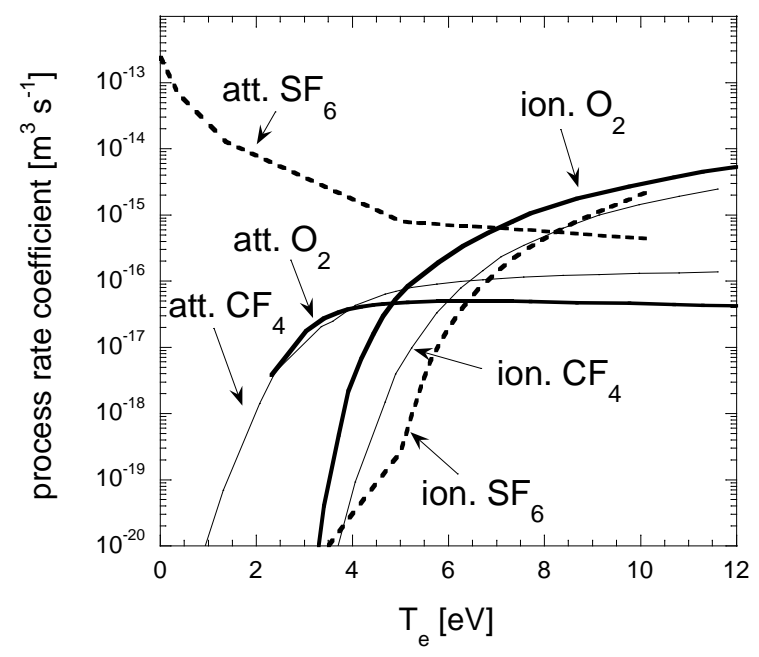

Figure 7. Calculations of attachment and ionization rate coefficients for $\mathrm{O}_{2}, \mathrm{CF}_{4}$ and $\mathrm{SF}_{6}$

that the two instability conditions described previously can be satisfied. On the contrary, $\mathrm{SF}_{6}$ plasma always remains stable. The attachment is much more effective in particular for low energy electrons, which are dominant by far. This particular attachment cross section results in low electronic density, experimentally confirmed by a very weak plasma luminosity and a low self-bias (only a few volts). Furthermore, the attachment rate coefficient decreases with $T_{e}$, which is why $R$ is negative at any electronic temperature. The attachment cross section is the source of the strong electronegativity and leads to the stability of $\mathrm{SF}_{6}$ discharges.

Knowing the corresponding attachment and ionization cross sections, this approach can be applied to other electronegative gases used in industry. For example, the cross 


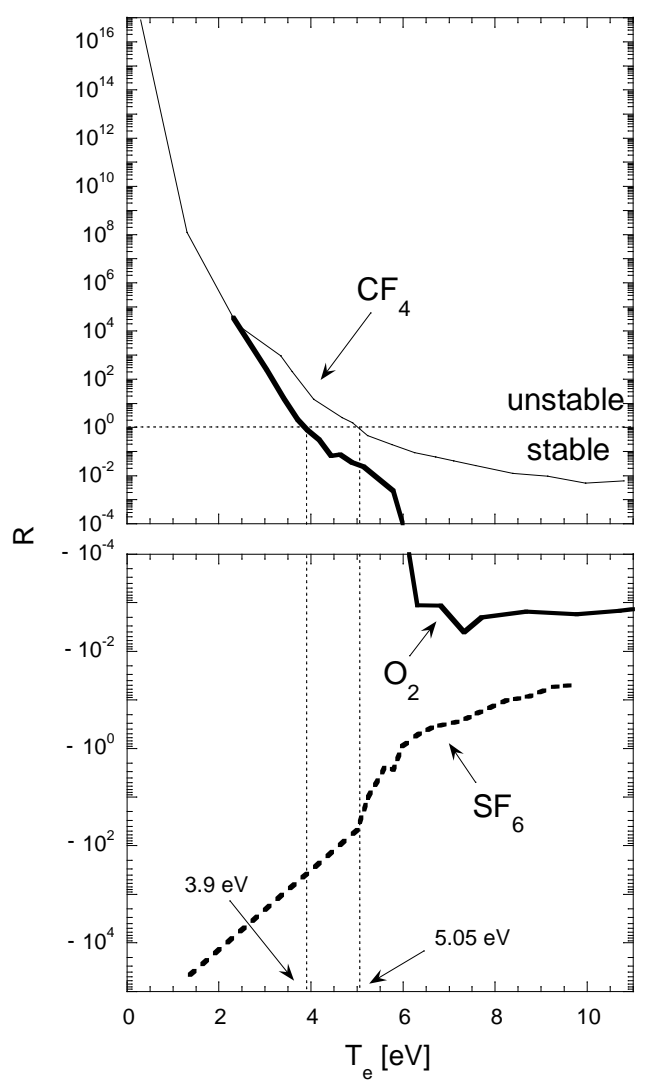

Figure 8. Instability criterion: calculation of the ratio $R$ for $\mathrm{O}_{2}, \mathrm{CF}_{4}$ and $\mathrm{SF}_{6}$. The plasma can be unstable if $T_{e}$ is lower than $3.9 \mathrm{eV}$ in $\mathrm{O}_{2}, 5 \mathrm{eV}$ in $\mathrm{CF}_{4}$

sections of $\mathrm{C}_{2} \mathrm{~F}_{6}[14,17], \mathrm{C}_{3} \mathrm{~F}_{8}[14,18]$ and $\mathrm{Cl}_{2}$ [19] indicate that these plasmas can be unstable, whereas $\mathrm{CCl}_{2} \mathrm{~F}_{2}$ [20] and $\mathrm{CF}_{3} \mathrm{I}$ [21] plasmas should remain stable. This remains to be confirmed experimentally.

Once the threshold temperature at which $R=1$ is known, a discharge simulation is useful to calculate the corresponding pressure and power conditions. These conditions can be compared with our experimental zones of instability. To simulate the discharge, we use Siglo-RF from Kinema Software [22], a fluid model solver. The discharges are certainly more complicated than those predicted by this model, but simulations give interesting qualitative informations. The chosen plasma parameters are the same as in our experiments. Figure 9 shows computation of $T_{e}$ as a function of pressure (at $V_{p p}$ $250 \mathrm{~V}$ ) and figure 10 shows $T_{e}$ as a function of $V_{p p}$ (at $0.25 \mathrm{mbar}$ ) for $\mathrm{O}_{2}$ and $\mathrm{CF}_{4}$.

At $250 \mathrm{~V}$, the simulation predicts unstability over $0.175 \mathrm{mbar}$ in $\mathrm{CF}_{4}$ (experimentally 0.1 mbar), and over 0.3 mbar in $\mathrm{O}_{2}$ (experimentally $0.195 \mathrm{mbar}$ ). Although the threshold pressures do not match exactly, the computed dependence of $T_{e}$ with the pressure is in good agreement with our results. The temperature decreases with increasing pressure, leading to stability of the discharge at low pressure. In practice, the attachment-induced ionization instability should not be a problem for etching applications, which are carried out at low pressure. 


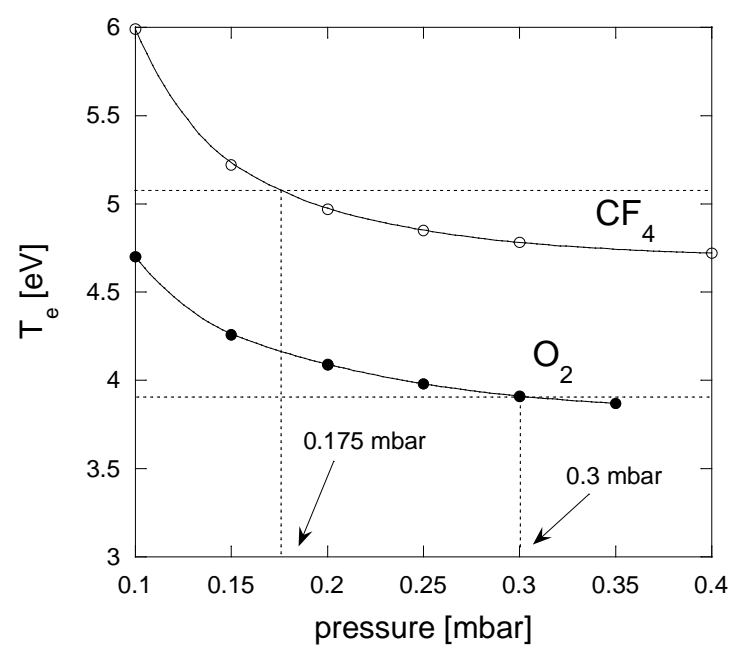

Figure 9. Electronic temperature vs pressure in $\mathrm{O}_{2}$ and $\mathrm{CF}_{4}$ calculated with Siglo- $\mathrm{RF}$ $\left(250 \mathrm{~V} V_{p p}\right)$. According to figure 8, the simulation predicts unstability over $0.175 \mathrm{mbar}$ in $\mathrm{CF}_{4}$ and over $0.3 \mathrm{mbar}$ in $\mathrm{O}_{2}$

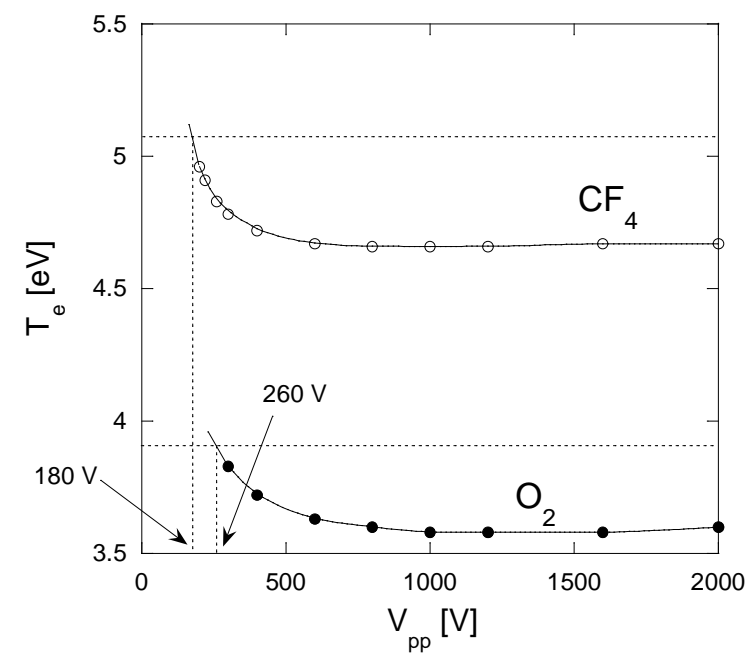

Figure 10. Electronic temperature vs $\mathrm{RF}$ voltage in $\mathrm{O}_{2}$ and $\mathrm{CF}_{4}$ calculated with Siglo-RF ( $0.25 \mathrm{mbar})$. According to figure 8 , the simulation predicts instability above $180 \mathrm{~V}$ in $\mathrm{CF}_{4}$ and above $260 \mathrm{~V}$ in $\mathrm{O}_{2}$

At 0.25 mbar, the computed zone of instability begins at $180 \mathrm{~V}$ in $\mathrm{CF}_{4}$ (experimentally between 220 and $550 \mathrm{~V}$ ), and at $260 \mathrm{~V}$ in $\mathrm{O}_{2}$ (experimentally between 185 and $490 \mathrm{~V}$ ). The lower thresholds are again not so far from our measurements. But the simulation does not predict an upper threshold as we measure it. In any case, the calculated temperature increase slightly at high power, but insufficiently to overcome $3.9 \mathrm{eV}$ and $5.05 \mathrm{eV}$ in $\mathrm{O}_{2}$ and $\mathrm{CF}_{4}$ respectively. 


\section{CONCLUSION}

The attachment-induced ionization instability occurs in $\mathrm{O}_{2}$ and $\mathrm{CF}_{4}$ capacitively coupled $\mathrm{RF}$ discharges under specific conditions of power and pressure, but not in $\mathrm{SF}_{6}$. The instability is experimentally observed through oscillations in the $\mathrm{kHz}$ range on electrical potentials $\left(V_{p p}, V_{s b}, V_{f}\right.$ and $\left.V_{p}\right)$, emission intensity and positive ion flux measurements. We also have obtained an experimental proof of the correlation between the instability and negative ions, with the measurements in diluted $\mathrm{Ar}+\mathrm{CF}_{4}$ and $\mathrm{Ar}+\mathrm{O}_{2}$ discharges. The cause of all these oscillations is coupled variations of $n_{e}$ and $T_{e}$. If the attachment rate coefficient increases more strongly than the ionization rate coefficient with $T_{e}$ and if electronic and negative ions densities are of the same order of magnitude, a small increase in $n_{e}$ can rapidly grow via a decrease in $T_{e}$. The plasma becomes unstable, and electronic and negative ions densities increase alternately.

$\mathrm{SF}_{6}$ differs from $\mathrm{O}_{2}$ and $\mathrm{CF}_{4}$ by its very high attachment cross section at low energy and by the negative dependence of this attachment rate coefficient. This results in strong electronegativity and stability of $\mathrm{SF}_{6}$ discharges. This experimental result is confirmed by the application of the theoretical instability criterion.

Simulation results confirm that the $\mathrm{O}_{2}$ and $\mathrm{CF}_{4}$ discharges are stable at low pressure and low power, because of a high electronic temperature. The calculated thresholds values are of the same order of magnitude as the experimental measurements, but the criterion gives a rather qualitative indication of plasma stability. It is difficult to determine precisely the unstable zone in terms of power and pressure by calculation. Nevertheless, this criterion can be applied to other electronegative gases if the ionization and attachment cross sections are known.

\section{References}

[1] Lieberman M A and Lichtenberg A J 1994 Principles of plasma discharges and materials processing (New York: Wiley)

[2] Ishikawa I, Matsumoto M and Suganomata S 1984 J. Phys. D: Appl. Phys. 1785

[3] Haas R A 1973 Phys. Rev. A 81017

[4] Nighan W L, Wiegand W J and Haas R A 1973 Appl. Phys. Let. 22579

[5] Nighan W L and Wiegand W J 1974 Phys. Rev. A 10922

[6] Tuszewski M 1996 J. Appl. Phys. 798967

[7] Chabert P, Lichtenberg A J, Lieberman M A and Marakhtanov A M 2001 Plasma Sources Sci. Technol. $\mathbf{1 0} 478$

[8] Katsch HM, Goehlich A, Kawetzki T, Quandt E and Döbele HF 1999 Appl. Phys. Let. 752023

[9] Gogolides E, Stathakopoulos M and Boudouvis A 1994 J. Phys. D: Appl. Phys. 271878

[10] Metsi E, Gogolides E and Boudouvis A 1996 Phys. Rev. E 54782

[11] Howling A A, Sansonnens L, Dorier J-L and Hollenstein C 1994 J. Appl. Phys. 751340

[12] Lawton S A and Phelps A V 1978 J. Chem. Phys. 691055

[13] Christophorou L G, Olthoff J K and Rao M V V S 1996 J. Phys. Chem. Ref. Data 251341

[14] Christophorou L G and Olthoff J K 1999 J. Phys. Chem. Ref. Data 28967

[15] Christophorou L G and Olthoff J K 2000 J. Phys. Chem. Ref. Data 29267

[16] Pitchford L C, O'Neil S V and Rumble J R 1981 Phys. Rev. A 23294

[17] Christophorou L G and Olthoff J K 1998 J. Phys. Chem. Ref. Data 271 
[18] Christophorou L G and Olthoff J K 1998 J. Phys. Chem. Ref. Data 27889

[19] Christophorou L G and Olthoff J K 1999 J. Phys. Chem. Ref. Data 28131

[20] Christophorou L G, Olthoff J K and Wang Y 1997 J. Phys. Chem. Ref. Data 261205

[21] Christophorou L G and Olthoff J K 2000 J. Phys. Chem. Ref. Data 29553

[22] Boeuf J-P and Pitchford L C 1995 Phys. Rev. E 511376 